\title{
Modelación matemática en la construcción conceptual de las inecuaciones al comparar magnitudes: uso de una norma medioambiental en la música
}

\author{
Mathematical modelling in the conceptual construction of inequalities when comparing \\ magnitudes: use of an environmental standard in music
}

LEMUS-CORTEZ, Neemias $^{1}$

ESCOBAR, Hugo ${ }^{2}$

\begin{abstract}
Resumen
La Modelación Matemática en el proceso de enseñanza-aprendizaje permite construir conceptos como las inecuaciones realizando comparaciones entre magnitudes. En el ciclo utilizado, se estudian tipos de argumentaciones que emergen desde la comparación y el uso del estilo de pensamiento matemático Visual-Analítico, produciéndose una unión entre las representaciones y cómo estas son interpretadas. La tarea construida fue desarrollada por estudiantes de último año de secundaria, cuyos resultados muestran cómo ellos construyen su conocimiento desde la sistematización de los conceptos.

Palabras clave: modelación matemática, argumentación, inecuación, comparar.
\end{abstract}

\begin{abstract}
Mathematical modelling in the teaching-learning process, allows the construction of concepts such as the inequalities making comparisons between magnitudes. In the cycle used, type of arguments are studied, that emerge from comparison and use of the mathematical visual-analytic thinking style, producing a union between the representations and how they are interpreted. The task was developed by senior in high school, whose results show how they can build their knowledge from systematization of concepts.
\end{abstract}

key words: mathematical modelling, arguments, inequality, compare.

\section{Introducción}

El estudio de las inecuaciones ha cobrado gran importancia en la enseñanza-aprendizaje de las matemáticas, debido a su influencia en la construcción de la teoría matemática (Halmaghi \& Liljedahl, 2012; Bernardis, Nitti \& Scaglia, 2017), como por ejemplo, la definición de límite dada por Cauchy (Borello, 2010), haciendo uso de los conceptos de comparar y acotar. Las inecuaciones han tenido un crecimiento gradual: estando presente a principios del desarrollo del conocimiento humano (Bagni, 2005), desarrollándose como una herramienta al

\footnotetext{
${ }^{1}$ Magíster en Didáctica de la Matemática y Profesor de Matemática. Departamento de matemática. Colegio Don Bosco. neemias.lemus@colegiodonbosco.cl

${ }^{2}$ Magíster en Didáctica de la Matemática y Profesor de Matemática. Programa de Acceso Efectivo y Acompañamiento a la Educación Superior (PACE) . Universidad de Playa Ancha. hugo.escobar@upla.cl
} 
servicio de la teoría matemática (Halmaghi \& Liljedahl, 2012) y llegando a convertirse en una disciplina de estudio (Bernardis et al., 2017).

En la revisión del estado del arte se ha identificado que las inecuaciones son un conocimiento tranversal (Bernardis et al., 2017), presente en una multiplicidad de conceptos: expresar condiciones (Bagni, 2005), comparar y tomar decisiones (Monje, Seckel \& Breda, 2018), donde la comparación está ligada a la relación de orden en $\mathbb{R}$ (Borello, 2010).

Halmaghi \& Liljedahl (2012), establecen que el estudiar la historia de los conceptos matemáticos ayuda a tener un éxito en el desarrollo del aprendizaje. Los autores realizaron un barrido histórico, mostrando que los matemáticos de la antigüedad eran expertos en comparar magnitudes y expresar relaciones entre ellos. Además, determinaron que los símbolos utilizados actualmente en las inecuaciones, eran usados sólo para comparar y no estaba al servicio de la aritmética.

En la enseñanza tradicional, no se evidencia la construcción del conocimiento de las inecuaciones lineales a través de enlaces entre aspectos conceptuales, como es el caso de comparar elementos. Esto queda demostrado, mediante el cómo se enseñan las inecuaciones en Chile (Muñoz, Gutiérrez \& Muñoz, 2016), sobre las bases que tienen los profesores para realizar sus clases (MINEDUC, 2015), y también, investigaciones que dan cuenta de este problema, el cual consiste en focalizar la enseñanza-aprendizaje de las inecuaciones lineales en procesos algorítmicos repetitivos previamente establecidos (Gatica \& Maz, 2012; De Souza, Nogueira de Lima \& Campo, 2015).

A partir de lo anterior y considerando el curriculum chileno, específicamente en el libro que el Ministerio de Educación de Chile (MINEDUC) entrega a los establecimientos educacionales hasta estos últimos años (Muñoz et al., 2016), se puede evidenciar la problemática descrita anteriormente, focalizada en las operaciones aritméticas que permiten encontrar la solución. En reiterados momentos (Tres veces en tres planas), como se muestra en la Figura 1, aparece la pregunta “¿Cómo hacerlo?”, ejemplificando la manera de cómo el estudiante debe resolver una inecuación lineal. Esta forma de presentar cómo se desarrolla el objeto matemático, produce en el estudiante dificultades en el aprendizaje por ausencia de significados (Garrote, Hidalgo \& Blanco, 2004, Gatica \& Maz, 2012) y por representar de forma aislada las diferentes representaciones de las inecuaciones (De Souza et al., 2015).

Figura 1

Ejemplo de ejercicio

\section{¿Cómo hacerlo?}

Si un joven es 22 años menor que su padre y 48 años menor que su abuelo, ¿a partir de qué edad la suma de los años que tienen él y su padre será mayor que la edad de su abuelo?

Si definimos como $x$ la edad del joven, entonces la edad de su padre y su abuelo serán $x+22$ y $x+48$, respectivamente. Luego, planteamos la inecuación:

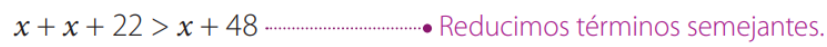

$2 x+22>x+48$ ……................... Restamos $x$.

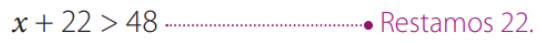

$x>26$

En consecuencia, si el joven es mayor de 26 años, la suma de su edad con la de su padre superará la cantidad de años que tiene su abuelo.

Fuente: Muñoz et al. (2016, p. 47)

La estructura de enseñanza-aprendizaje propuesta en la figura presentada, solo fomenta el aprendizaje de algoritmos que se aprenden de forma memorística. En este sentido, el lenguaje natural y las gráficas son consideradas como elementos o herramientas de carácter secundario al desarrollo algebraico para la resolución 
de inecuaciones lineales, produciendo dificultades adicionales (De Souza et al., 2015) y no dando lugar a la argumentación (Cardetti \& LeMay, 2019).

Se identifica la problemática de la enseñanza-aprendizaje de las inecuaciones lineales, en el énfasis puesto en la resolución algebraica y no en los conceptos ni en las representaciones gráficas, produciendo escasas situaciones didácticas para construir conceptualmente la conexión entre la matemática y la realidad de los estudiantes. Por lo tanto, no se hace uso de la argumentación como medio para fortalecer la comprensión conceptual del objeto matemático. Gatica y Maz (2012) confirman lo descrito anteriormente, indicando que los estudiantes no aprenden las inecuaciones desde los aspectos conceptuales, sino al contrario, desarrollan su aprendizaje desde lo procedimental y a través de reglas y algoritmos repetitivos.

Las investigaciones muestran que el aprendizaje de las inecuaciones lineales debe realizarse mediante la conexión de las diferentes representaciones que posee el objeto matemático (Barbosa, 2003; Hernández, 2013; Torres, 2013; De Souza et al., 2015), para que a través de ellas se realice un proceso argumentativo como herramienta para apoyar el razonamiento matemático (Cardetti \& LeMay, 2019). Por lo tanto, se establece que mediante la Teoría de Registro de Representaciones Semióticas (Duval, 1995) existen diferentes registros de representaciones, que en el caso de las inecuaciones son: lenguaje algebraico, gráfico, tabular y natural.

La Modelación Matemática viene a realizar una conexión entre la realidad del estudiante y las matemáticas, debido a que metafóricamente es un puente entre saberes (Freid \& Bergsten, 2019). Se identifica un puente entre lo que el estudiante no conoce y el uso que el estudiante puede realizar con este conocimiento, mediante el concepto de comparar. En esta conexión se materializan usos de la argumentación, debido a que por medio de ella se generan relaciones entre representaciones y entre conocimientos previos con el nuevo conocimiento (Cardetti \& LeMay, 2019).

Esta investigación exploratoria no correlacional, pretende analizar el tipo de argumentación que emplean los estudiantes al momento de resolver una tarea de modelación y de una forma más específica: se analizarán, describirán e interpretarán las producciones materializadas en las tareas de modelación. A la luz de los resultados obtenidos mediante la sistematización de los conceptos, se dará respuesta a nuestra interrogante: ¿Qué tipo de argumentaciones permite una tarea de modelación en la construcción conceptual de inecuaciones lineales?

\section{Marco conceptual}

La Modelación Matemática posee diferentes ciclos de modelación, pero para nuestra investigación utilizaremos el ciclo de modelación de Blum-Borromeo (Borromeo-Ferri, 2010), que se caracteriza por tener una perspectiva más cognitiva y que nace desde el ciclo propuesto por Blum \& Leiß (2005).

Firgura 2

Ciclo de Modelación Blum-Borromeo

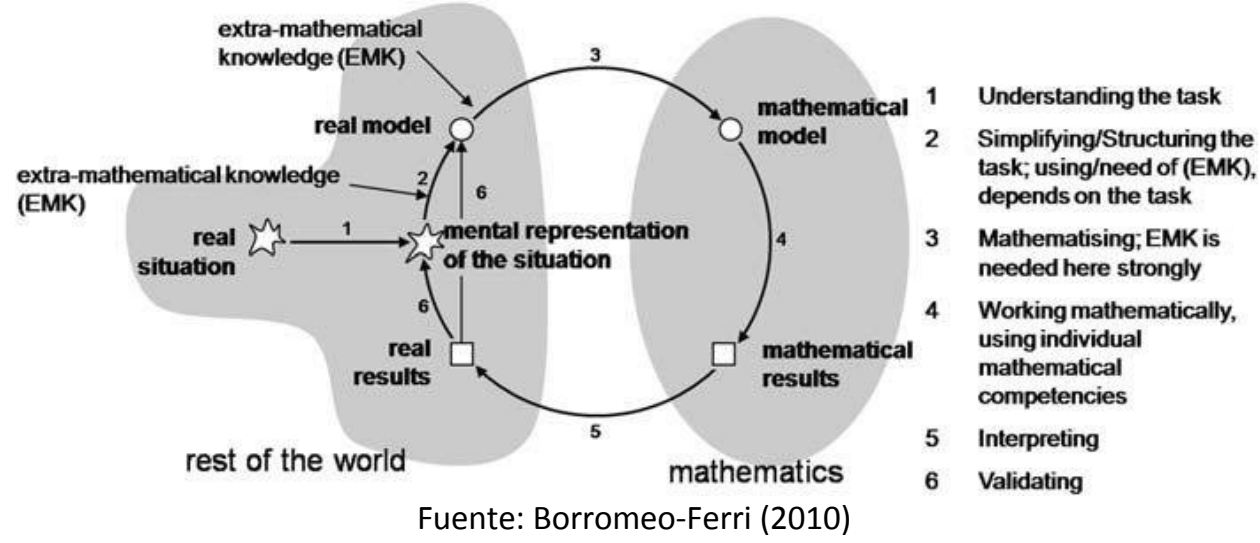


Freid \& Bergsten (2018) establecen que la modelación matemática debe ser una aproximación entre el saber matemático del estudiante y las inecuaciones, específicamente para nuestro caso. Los autores determinaron que metafóricamente existe un puente entre las matemáticas y la realidad, relación que se pude llevar a cabo mediante el concepto de comparación inherente en las inecuaciones. Borello (2010) en su estudio determinó que: "En nuestra vida cotidiana comparamos y acotamos continuamente ya que estas actividades son las que nos permiten emitir juicios y, por consecuencia, tomar decisiones" (p. 47).

Según la Figura 2, los estudiantes reciben una tarea que tiene un contexto real (DJ en una fiesta) y donde existe un fenómeno a modelar (Decibeles de una canción). La tarea se debe entender en el mundo del estudiante para poder estructurar un modelo real de la situación, que en nuestro caso está dado por la comparación entre las representaciones gráficas de los decibeles de la canción, en relación con el tiempo y las normas de emisión de ruidos molestos generados por fuentes fijas (Ley № 19300, 2012). Desde este modelo se realiza una matematización que ayudará a los estudiantes a construir un modelo matemático y que este sea válido. A través del trabajo vertido en cada fase, se debe hacer uso de competencias matemáticas para obtener resultados matemáticos, cuyos resultados se muestran en intervalos en la recta real. Estos resultados son interpretados para obtener una causa real del modelo, produciendo una representación mental de la situación (BorromeoFerri, 2010).

La perspectiva cognitiva que tiene el ciclo de modelación presentado, permite analizar los procesos cognitivos que se desarrollan en el ciclo y como se comprenden, pues los usos de modelos como imágenes mentales (representaciones) ayuda a los procesos cognitivos, como la abstracción o la generalización (Kaiser \& Sriraman, 2006).

Según Borromeo-Ferri (2010) los procesos cognitivos provienen de las representaciones mentales de las situaciones, vinculándose con la teoría de los estilos de pensamiento matemático. La autora agrega que el término estilo de pensamiento matemático, es cuando un estudiante prefiere presentar, comprender y pensar a través de hechos matemáticos y conexiones, utilizando ciertas imaginaciones internas. Además, se definen tres estilos de pensamiento matemático para la ruta de modelado: pensamiento visual, pensamiento analítico y pensamiento integrado.

Focalizado en el estilo de pensamiento matemático integrado, Zazkis, Dubinsky \& Dautermann (1996), interiorizaron sobre los estilos de pensamiento matemático, enfocándose en la visualización y el análisis, proponiendo un modelo alternativo (visualizador/analizador o V/A) que determina que estos dos estilos de pensamiento matemático son mutuamente dependientes en la resolución de problemas matemáticos. Asumiendo que estos dos estilos al estar presentes e integrados, permite construir una comprensión rica de los conceptos matemáticos.

Zazkis et al. (1996) declaran que la visualización o pensamiento visual consiste en el hacer y no en el producto de lo que se hace, radicando en la conexión entre la imagen y lo mental. Para el caso del pensamiento analítico, los autores ejemplifican este tipo de pensamiento con un biólogo que estudia la naturaleza de una planta, que al descomponerla da lugar a la relación de sus partes y sistematizándolas en otras relaciones.

Para esta investigación se hace necesario posicionar al pensamiento matemático integrado como un agente de entrada para recorrer la ruta del modelado, pues al momento que los estudiantes hacen uso del concepto de comparar en la tarea de modelación, actuarán en base a los elementos visuales y analíticos presentes en la situación. 


\subsection{La argumentación}

La argumentación que se produce en estos procesos cognitivos es de mucha importancia, pues la argumentación lógica es una habilidad necesaria en el aula, debido a que muestra el carácter de verdad de una proposición (Crespo, 2005, Cardetti \& LeMay, 2018). De Gamboa, Planas y Edo (2010) determinan que esta habilidad ayuda a comprender y producir una relación de justificación entre proposiciones. Además, Crespo (2005) muestra que no es sólo una riqueza en el dominio de la tarea presentada, sino en el razonamiento de las tareas futuras y con distinto origen.

Las bases curriculares buscan que los estudiantes sean personas capaces de razonar y ser críticos, pero esto sólo se puede desarrollar a través de prácticas argumentativas, donde aprenda a reconocer argumentos válidos. En el caso de Chile, la argumentación es una de las habilidades que se espera ser desarrollada en los estudiantes mediante tareas de modelación (MINEDUC, 2019).

En la argumentación de forma global, León y Calderón (2001) identificaron dos tipos de recursos argumentativos: matemáticos y discursivos. Estos recursos están inmersos en la argumentación como complemento de la validación. Para ello, los autores determinaron tres tipos de fases que construyen el proceso argumentativo global: De formulación, de confrontación y análisis y consolidación de argumentos.

Cardetti \& LeMay (2019) en el proceso argumentativo de una tarea matemática, identificaron y desarrollaron cinco categorías de argumentación:

1. Dar sentido a los procedimientos: Consiste en los procedimientos que los estudiantes deben hacer desde sus explicaciones y que no sean determinadas por un algoritmo preestablecido.

2. Analizar conceptos erróneos: El error juega un rol protagónico en la comprensión conceptual, ya que el poner atención a los errores y sus correcciones, beneficia la argumentación y la comprensión de un concepto.

3. Vincular conceptos: El estudiante vincula diferentes conceptos matemáticos, temas y procesos que, al estar juntos, proporciona una relación entre diferentes ideas, provocando una comprensión más profunda de las matemáticas en su conjunto.

4. Conexión entre conocimientos previos: Los conocimientos previos y su importancia, se debe a que al argumentar el estudiante organiza y reorganiza estos conocimientos previos para lograr nuevas comprensiones conceptuales.

5. Conexión entre representaciones: Alude al manejo de representaciones por parte del estudiante, pues manejar diferentes representaciones en relación con un tema y las distintas diferencias entre representaciones, caracteriza el progreso del entendimiento de un concepto.

Las categorías presentadas, serán de mucha ayuda al analizar las argumentaciones de los estudiantes en el ciclo de modelación, ya que para efectos de la investigación nos vamos a enfocar en las fases tres, cuatro y cinco (Figura 2). Reconocemos que en cada fase del ciclo de modelación existen tipos de argumentaciones, pero por causa de nuestro análisis, sólo nos centraremos en estas tres fases.

El estudiante al enfrentarse a la tarea de modelación y transitar por cada fase del ciclo, desarrolla cada fase según sus características. Al tener el modelo de la situación real, los estudiantes utilizan gráficas que permiten el desarrollo del estilo de pensamiento matemático integrado como agente de entrada y agente transversal, pues emplearán la comparación que es un concepto trascendental en las inecuaciones. Por lo tanto, la argumentación como herramienta de apoyo para el razonamiento matemático, permitirá esta relación de justificación entre proposiciones, emergiendo desde todo este trabajo: Dar sentido a los procedimientos, analizar conceptos erróneos, vincular conceptos, conexión entre conocimientos previos y conexión entre representaciones. 


\title{
3. Metodología
}

Esta investigación exploratoria no correlacional e investigación-acción, responde a la existencia de una problemática, desde la cual se producen estrategias de acción y una actuación participativa de los involucrados y, de esta forma, desarrollar una comprensión sistemática y profunda de las situaciones que, al ejecutarse y evaluarse, finaliza en un producto (reflexión y acción) que da solución a la problemática identificada (Kemmis \& McTaggart, 2013). Además, la recogida de datos de la tarea de modelación se realizó mediante la producción de los estudiantes y videos del plenario de la clase.

\subsection{Unidad de análisis}

Los sujetos de estudio son estudiantes del nivel de cuarto año de enseñanza media, con una edad de 17 a 18 años, siendo 13 mujeres y 15 hombres. Los grupos de trabajo que realizaron la tarea fueron 7, quienes trabajaron de forma cooperativa resolviendo cada una de las interrogantes planteadas. Los estudiantes pertenecen a un colegio particular subvencionado de la Región del Libertador Bernando O'Higgins. El colegio es científico humanista. Además, los participantes no conocían la tarea de modelación y no fueron instruidos en el cómo desarrollar la tarea, con la intención de evidenciar de forma más natural las respuestas y que estas no estén intencionadas por un conocimiento previo.

\subsection{Tarea de modelación}

Para esta investigación se construyó una tarea de modelación, desde un contexto de actualidad, haciendo uso de la música y de un sonómetro (App). A continuación, en la Figura 3, se detalla la tarea de modelación presentada a los estudiantes.

\section{Figura 3}

Tarea de modelación

\begin{abstract}
La emisión del sonido; como la voz, el canto de las aves y ruido ambiente; puede ser analizado de varias formas. Una forma es utilizar el sonómetro, que captura la intensidad del sonido de las ondas sonoras, mostrando una gráfica y de esta manera poder tener un medio para el estudio del sonido. El Decreto 38 del Ministerio del Medio Ambiente (norma de emisión de ruidos molestos generados por fuentes fijas) entrega los niveles máximos permitidos de emisión sonora generados por fuentes fijas para la comunidad. Dispone que el ruido máximo para las zonas residenciales es de 55 decibeles $(d b)$ en horario de 07 a 21 horas.

Para realizar nuestra tarea, leamos atentamente la siguiente situación: "Arturo esta de cumpleaños y realiza una fiesta en su casa, contratando un dj que desea partir la fiesta a las 19 horas con la canción despacito de Luis Fonsi, a un volumen moderado. Al cabo de un minuto, Arturo se acerca al Dj y muy molesto le dice que la música está despacio y que debe subirle..."

Si tú eres el fiscalizador y cuentas con las gráficas de la canción puesta por el DJ.

1. ¿En qué momentos la canción sobrepasa la norma del Ministerio del Medio Ambiente?

- subrayar con lápiz rojo el o los tramos en qué ocurre este fenómeno.

- representar en una recta real cada tramo detectado.

2. ¿Cuánto tiempo el DJ sobrepasó la norma?

3. Si fueses el inspector, ¿qué le dirías al DJ?
\end{abstract}

Fuente: Elaboración de los autores

En el momento de la implementación del instrumento y recogida de datos, se entrega la tarea (Figura 3) y seis láminas que contienen gráficas que miden los decibeles de la canción elegida, despacito (Ender, Fonsi \& Yankee, 2017) y, los datos entregados por el sonómetro. Cada lámina describe 10 segundos de la canción en una gráfica, en base a las variables tiempo y la intensidad del sonido, siendo en total 60 segundos a analizar de la canción. 


\subsection{Categorías de análisis}

Al tener un marco conceptual que se estructura principalmente entre la modelación matemática y la argumentación, se conforman las categorías de análisis de la siguiente manera: en primer lugar, están focalizadas en tres fases (fase 3, 4 y 5) del ciclo de modelación (Borromeo-Ferri, 2010) y, en segundo lugar, se encuentra las cinco categorías de argumentación dadas por Cardetti \& LeMay (2019). A continuación, se presenta la Tabla 1 con las categorías de análisis y sus respectivas descripciones.

Tabla 1

Categorías de análisis

\begin{tabular}{|c|c|c|c|}
\hline Tarea & $\begin{array}{l}\text { Fases del ciclo de } \\
\text { modelación }\end{array}$ & Argumentación & Descripción \\
\hline \multirow[t]{4}{*}{$\begin{array}{l}1 \text { ¿En qué } \\
\text { momento la } \\
\text { canción } \\
\text { sobrepasa la } \\
\text { norma del medio } \\
\text { ambiente? }\end{array}$} & \multirow[t]{2}{*}{$\begin{array}{l}\text { Matematización } \\
\text { (Fase 3) }\end{array}$} & $\begin{array}{l}\text { Conexión entre } \\
\text { conocimientos } \\
\text { previos }\end{array}$ & $\begin{array}{l}\text { Los estudiantes mediante las gráficas realizan una función constante } \\
\text { en } f(x)=55 \text { o una recta horizontal en } x=55 \text {, representando la } \\
\text { norma descrita en el decreto } 38 \text { del Ministerio del Medio Ambiente } \\
\text { (Ley № } 19300,2011 \text { ), cuyos conocimientos previos sobre función y } \\
\text { recta fueron aprendidos en cursos anteriores. }\end{array}$ \\
\hline & & $\begin{array}{l}\text { Conexión entre } \\
\text { representaciones }\end{array}$ & $\begin{array}{l}\text { Se identifican los momentos en que la curva de los decibeles } \\
\text { interseca a esta función constante o línea horizontal, produciendo } \\
\text { un conjunto de elementos que sobrepasa la línea horizontal y otros } \\
\text { que están bajo esa línea. Se realiza la conexión entre una } \\
\text { representación en un plano con la representación en una recta real. }\end{array}$ \\
\hline & \multirow[t]{2}{*}{$\begin{array}{l}\text { Trabajo } \\
\text { matemático (Fase } \\
\text { 4) }\end{array}$} & Vinculan conceptos & $\begin{array}{l}\text { Cada conjunto es transcrito como instantes en una recta real, } \\
\text { estableciéndose la recta real como el tiempo donde la canción } \\
\text { sobrepasa la norma. } \\
\text { Aquí se vincula el concepto de RECTA REAL-TIEMPO. Vinculando } \\
\text { conceptos matemáticos con conceptos físicos. }\end{array}$ \\
\hline & & $\begin{array}{l}\text { Conexión entre } \\
\text { representaciones }\end{array}$ & $\begin{array}{l}\text { Este conjunto de momentos descritos en una recta real, son } \\
\text { transformados en intervalos de tiempo, específicamente intervalos } \\
\text { de tiempos en que la canción sobrepasa a la norma. } \\
\text { Aquí se realiza la conexión entre la representación gráfica de los } \\
\text { momentos con una representación algebraica y proposicional de } \\
\text { intervalos. }\end{array}$ \\
\hline $\begin{array}{l}2 \text { ¿Cuánto } \\
\text { tiempo el DJ } \\
\text { sobrepasó la } \\
\text { norma? }\end{array}$ & $\begin{array}{l}\text { Interpretación de } \\
\text { los resultados } \\
\text { (Fase } 5 \text { ) }\end{array}$ & $\begin{array}{l}\text { Analizar conceptos } \\
\text { erróneos }\end{array}$ & $\begin{array}{l}\text { Al momento de realizar las conexiones entre representaciones } \\
\text { (gráfica de la recta con los intervalos) pueden incurrir en error de } \\
\text { escritura y errores de comprensión de un intervalo. Al comparar } \\
\text { resultados con otros pueden surgir argumentaciones sobre la } \\
\text { construcción y medición de los intervalos. }\end{array}$ \\
\hline \multirow[t]{3}{*}{$\begin{array}{l}3 \text { Si fueras } \\
\text { inspector ¿Qué le } \\
\text { dirías al DJ? }\end{array}$} & \multirow[t]{3}{*}{$\begin{array}{l}\text { Interpretación de } \\
\text { los resultados } \\
\text { (Fase 5) }\end{array}$} & $\begin{array}{l}\text { Dar sentido a los } \\
\text { procedimientos }\end{array}$ & $\begin{array}{l}\text { Los estudiantes realizan una interpretación de los intervalos en } \\
\text { relación con la medición total del tiempo la que requiere de dos } \\
\text { acciones cognitivas: }\end{array}$ \\
\hline & & & $\begin{array}{l}\text { 1) Comprender que el tiempo asociado a un intervalo se puede } \\
\text { obtener a partir de la diferencia entre los límites de los } \\
\text { intervalos. Diferencia que deberá considerar una sustracción } \\
\text { en un sistema sexagesimal como lo es el de nuestro sistema } \\
\text { horario. }\end{array}$ \\
\hline & & & $\begin{array}{l}\text { 2) Acumular o sumar todas las mediciones de tiempo obtenidas } \\
\text { para cada uno de los intervalos que cumplan con el criterio, } \\
\text { teniendo en cuenta también que la suma debe realizarse bajo } \\
\text { la lógica del sistema horario. } \\
\text { Se da sentido a todo el desarrollo realizado, cuando se realiza la } \\
\text { interpretación de los resultados en el mundo real. }\end{array}$ \\
\hline
\end{tabular}

Fuente: Elaboración de los autores 
Mediante estas categorías de análisis construidas se analizarán los resultados, observando si estas categorías se evidencian en la ruta de modelación en las diferentes fases a observar y cómo las argumentaciones son constructoras de conocimiento matemático.

Dada las características de esta tarea de modelación, si bien es cierto las fases de modelación dan cuenta de cómo se va construyendo un concepto, se hace necesario explicitar que esta taxonomía de argumentación que se ha considerado fundamentalmente desde las fases de modelación, permite ver la matematización con fuerza en la argumentación.

\section{Resultados}

En este apartado se presentan algunas de las producciones de los estudiantes, mostrando aquellos resultados que son más relevantes y sintetizados, debido a lo extenso que fueron los datos recogidos. Cabe señalar que, de los siete grupos conformados para realizar la tarea, se expondrá la muestra más significativa de los datos (grupo 5 y 6), dejando claro que la gran mayoría, seis de los siete grupos, realizó procedimientos muy parecidos.

En la primera parte de la presentación de resultados, se mostrarán dos producciones de grupos diferentes. En esta parte, se ha decidido seleccionar una de las seis láminas de las gráficas de los decibeles de la canción, para ejemplificar el proceder de cada grupo y a través de esta muestra generalizar lo realizado por parte de los demás grupos. La segunda parte y hasta el final de la exhibición de los resultados, utilizaremos las producciones de los grupos 5 y 6 , con el fin de visualizar el proceso a analizar.

Inicialmente, los estudiantes deben realizar un procedimiento de subrayar con lápiz rojo el o los tramos en que la canción sobrepasa la norma, respondiendo a la pregunta: ¿En qué momento la canción sobrepasa la norma del Ministerio del Medio Ambiente?. Entonces, los estudiantes establecieron una línea horizontal en $x=55$.

\section{Figura 4}

Forma 1 de establecer la norma del Ministerio del Medio Ambiente

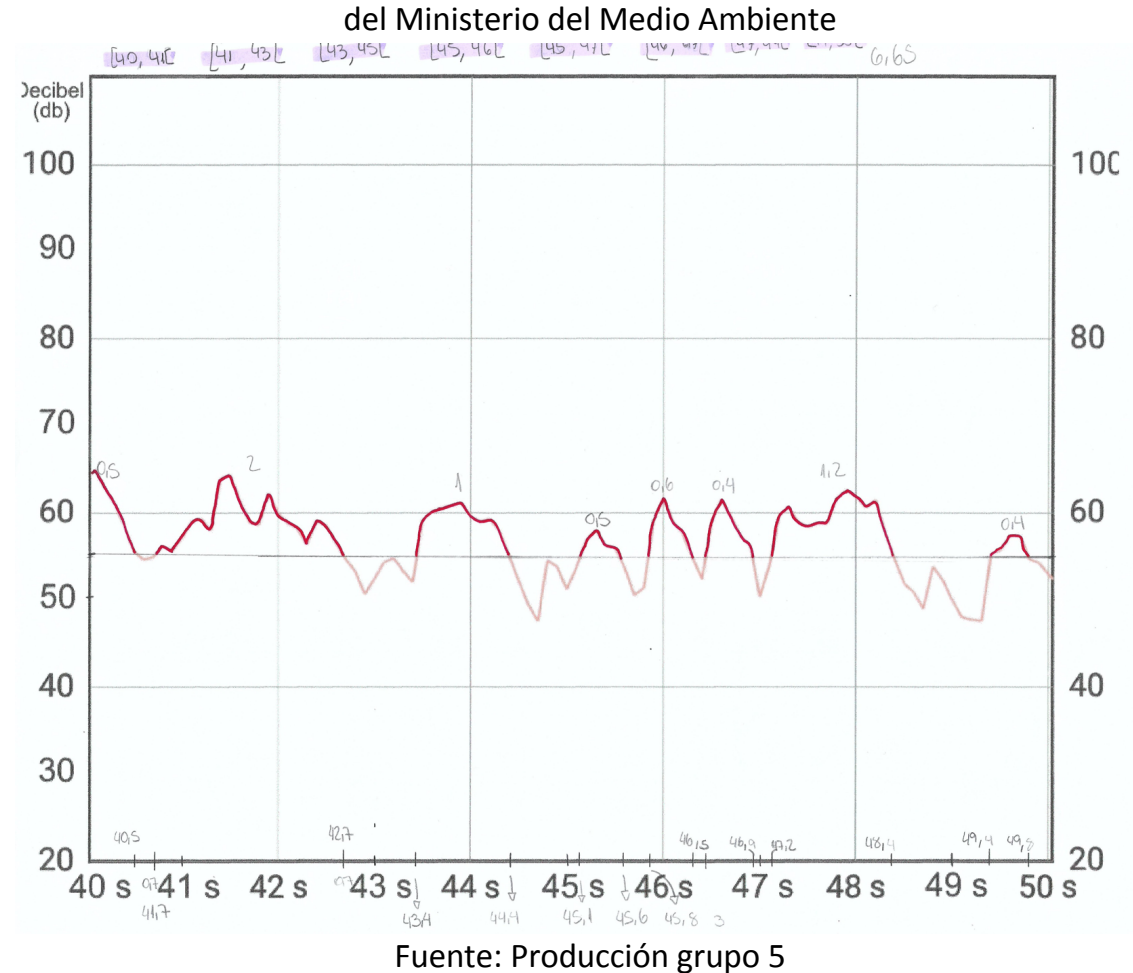

Fuente: Producción grupo 5 
La gran mayoría de los grupos procedieron en este momento como se muestra en la figura 4, sólo existe el grupo 7 que trazaron segmentos perpendiculares al eje del tiempo en segundos, como una estrategia para determinar los intervalos de tiempo en que la canción sobrepasó la norma, situación que se ejemplifica en la figura 5.

Todos los grupos en el instante de buscar cada momento en que la canción sobrepasó la norma del Ministerio del Medio Ambiente, desde los 55 decibeles, utilizaron gran parte del tiempo en buscar el cómo resolver lo preguntado, llegando a plasmar lo realizado en la figura 4 y 5.

Figura 5

Forma 2 de establecer la norma del Ministerio del Medio Ambiente

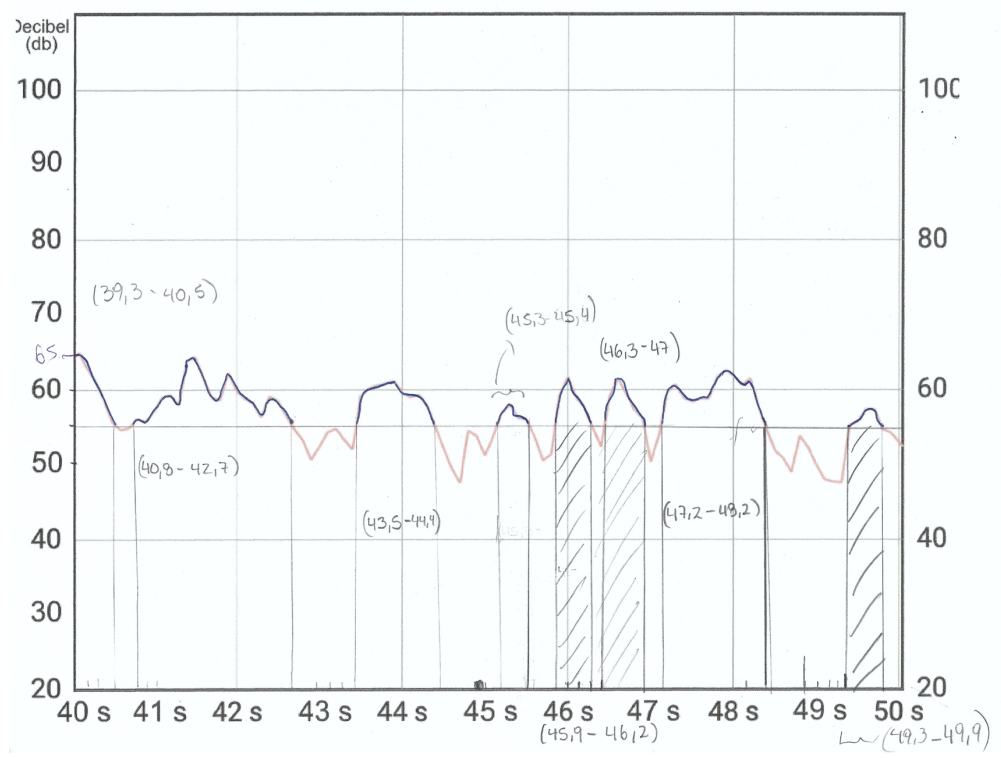

Fuente: producción grupo 6.

Luego de realizar la acción de establecer esta norma, los grupos debían proceder en representar en una recta real por cada tramo detectado. Seis de los siete grupos realizaron el procedimiento de plasmar en una recta real todos los momentos en que la canción sobrepasó la norma, como se muestra en la Figura 6 . El grupo cinco no realizó la recta real.

Figura 6

Recta real de los momentos en que la canción sobrepasa la norma

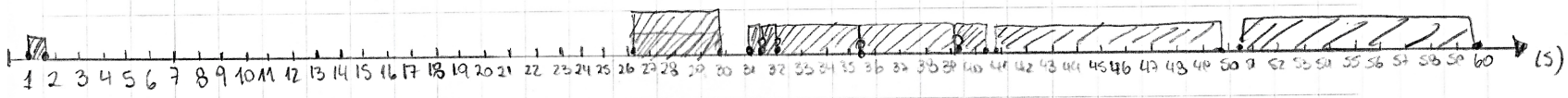

Fuente: Producción grupo 6

En relación a esto último, los estudiantes realizaron un procedimiento que no estaba previsto, que durante el proceso de confección de la recta real con cada momento en que la canción sobrepasó la norma, cuatro de los siete grupos, realizaron una organización de datos por momentos y esto se ve reflejado en la figura 7. 
Figura 7

Lista descriptiva de los momentos

observados por el grupo 6

Aer Tramo.

Se sobre pasa des de

$1^{\circ}-15$ hasta el $1,5 \mathrm{seg} . \quad[1 ; 1,5]$

2 do Tramo

Se sobre pasa disde

$1^{\circ}-26,3$ hasta 27,1 seg $\quad[26,3 ; 29,9]$

$2^{\circ}-27,8$ hasta e127,9

$3^{\circ}-28,3$ hasta el 29

$4^{\circ}-29,9$ hasta el $30,3 \mathrm{seg}$

Ber Tramo

Se sobre pasa desde

$1^{0}-31$ hasta el 31,2

$2^{\circ}-31,2$ hosta el 32

$3^{\circ}-32$ hasta el $35,3 \mathrm{seg}$

$4^{\circ}-35,3$ hasta el 39,3

$5^{\circ}-39,3$ hasta el 40,5

$[31 ; 31,2]$

$73,1,2 ; 32]$

] $32 ; 35,3]$

$335,3 ; 39,3]$

$339,3 ; 40,5]$ $4^{\text {to }}$ Tramo

Se sobre pasa desde

$1^{\circ}-40,8$ hasta 42,7

$2^{0}-43,5$ hasta el 44,4

$3^{\circ}-45,3$ hasta el 45,4

$4^{\circ}-45,9$ hosta el 46,2

$5^{\circ} 46,3$ hosta el $s^{47}$

$6^{\circ} 47,2$ hasta el 48,3

$7^{\circ} 49,3$ hasta el 49,9

5 to Tramo

Se sobre pasa desde

$1^{0}-50,5$ hasta el $55,4 \mathrm{seg} \quad[50,5 ; 60]$

$2^{\circ}-55,8$ hasta $59,8 \mathrm{seg}$

$3^{\circ}-59,9$ hasta el seg 60.

$[40,8 ; 49,9]$
Fuente: Producción grupo 6

El grupo 5 no realizó el resumen de los intervalos en una recta numérica, pues determinaron inmediatamente que el tiempo asociado a cada intervalo se puede obtener mediante la diferencia entre los límites de los intervalos. Esta situación se evidencia en la Figura 8.

Figura 8

Tiempo asociado a cada intervalo

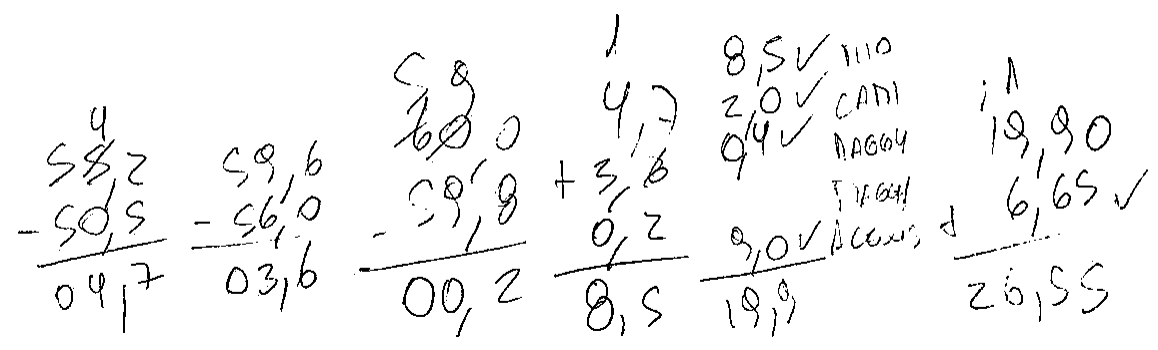

Fuente: Producción grupo 5

Luego de realizar el procedimiento de encontrar el tiempo asociado a cada intervalo, todos los grupos entregaron respuestas numéricas en relación a la pregunta: ¿Cuánto tiempo el DJ sobrepasó la norma? En general los valores entregados como respuesta, fueron entre los 26,55 y 31,5 segundos.

Finalmente, los estudiantes debían responder a la pregunta 3: Si fueras inspector ¿qué le dirías al DJ?, obteniendo diferentes respuestas: asociadas a multas, problemas auditivos y sentido común. A continuación, se muestra una de esas respuestas.

\section{Figura 9}

Respuesta a la pregunta 3

Que baje el volumen a un nivel tolerable ya que osta sobreposondo los sedb permitidos por la ley y puede recibir una multa

Fuente: Producción grupo 5 
Las respuestas realizadas a cada una de las interrogantes, determinan una contextualización y argumentación matemática, siendo la visualización en la matemática, un elemento importante para el desarrollo del concepto en el ciclo de modelación. La visualización comienza cuando se debe determinar la norma y establecer los momentos que la canción sobrepasa dicha norma. Luego, como producto de la visualización, viene un trabajo analítico con los datos entregados. Los estudiantes al tener elementos bajo la taxonomía argumentativa, pueden realizar argumentaciones en cada momento del ciclo.

\section{Discusión}

Los estudiantes al momento de recibir la tarea de modelación, demoraron en resolverla, por causa de que era la primera tarea de este estilo a la cual se enfrentaban. Esto se debe a que la modelación matemática no es común en las aulas, desde el año 2015 la modelación viene tomando fuerza en las bases curriculares como una habilidad (MINEDUC, 2015) en relación con los años anteriores. A fines del año 2019, el MINEDUC (2019) ha definido que la Modelación Matemática es un proceso que busca integrar las habilidades (representar, modelar, resolver problemas y argumentar y comunicar) y a través de este proceso tomar decisiones fundamentadas. Otra situación a la que se enfrentan los docentes es la falta de capacitación sobre esta área, porque aunque los esfuerzos de definir de buena forma la modelación matemática viene plasmados en las bases curriculares, existen múltiples creencias y definiciones erróneas en relación al tema en cuestión. En esta misma línea, Blomhøj (2019) declara que la modelación matemática está incluida en los programas de estudio, pero no está integradas en las aulas.

La argumentación tiene la misma falencia que la modelación matemática, aunque es transversal en todas las disciplinas, pero en matemática no es algo que tenga relevancia (Crespo, 2005; De Gamboa et al., 2010). La modelación está presente en las bases curriculares, así también la argumentación, siendo ambas consideradas por el Ministerio de Educación de Chile como dos de las cuatro habilidades a desarrollar en el aula, pues estas son protagonistas en la adquisición de nuevas destrezas y conceptos (MINEDUC, 2015). La argumentación es importante desde las bases de la educación, para los docentes de matemática no es de mucha importancia, mediante los resultados se puede evidenciar que para este grupo de estudiantes la argumentación puede ser promovida por tareas de modelación, pues los estudiantes muestran sus capacidades argumentativas al momento de responder a las interrogantes.

Por causa de las intenciones de esta investigación, como hemos dicho, sólo nos centraremos en lo que ocurre en las fases 3, 4 y 5 del ciclo de modelación, en relación con los tipos de argumentaciones presentados.

Al momento de enfrentarse los estudiantes a la tarea y a cada lámina del sonómetro con las mediciones de la canción, las estrategias utilizadas para resolver la tarea son similar a lo visto en la Figura 4. Los grupos no evocaron la idea de $\mathrm{f}(\mathrm{x})=55$ como función constante, pero si realizaron una recta horizontal haciendo alusión a lo que se pedía matemáticamente, lo cual es resultado de la matematización mediante la visualización. Los estudiantes argumentan a través de estas representaciones, pues realizan una conexión entre ellas, por causa de conocimientos previos que ellos tienen. Quizás la función constante que se buscaba identificar como tal, surgió de los estudiantes a través del pensamiento visual, porque estos conocimientos, que eran previos, ya estaban en su mente.

En referencia a lo anterior, la argumentación que relaciona la recta horizontal y la curva de la canción, provocó conexión entre representaciones. Las acciones plasmadas en las Figuras 4 y 5, muestran que existen intersecciones entre funciones, dando lugar a determinar comparaciones entre ellas. Las intersecciones según De Souza et al. (2015) se puede mirar como una comparación entre dos funciones. Entonces, las respuestas nacidas desde la matematización en base a la visualización, produjeron elementos que están reportados en la literatura. Luego, la conexión realizada entre representaciones tiene relación a esta comparación entre funciones 
y que al interceptarse existen puntos que delimitan esta comparación, evocando la existencia de dos tipos de representaciones, sobre la norma y bajo la norma.

Este tipo de representaciones emergentes, donde existen estos puntos que delimitan la comparación, definen los límites que representan los intervalos y que contienen los tiempos que la canción sobrepasó la norma.

Al comparar la recta horizontal y la curva de la canción no se hace uso de inecuaciones ni de su simbología, sino que se trabaja con el concepto inherente al objeto matemático que es comparar dos magnitudes. En consecuencia, y como se muestra en las Figuras 6 y 7, la identificación de dos tipos de representaciones, sobre la norma y bajo la norma, permite a los estudiantes trabajar inicialmente desde la construcción conceptual, ayudando al entendimiento de los procesos y a determinar el conjunto solución de la tarea sin la necesidad de realizar procesos algorítmicos.

Otra conexión entre representaciones identificada a través de la argumentación, la encontramos en el momento en que se detectaron los tramos en que la canción sobrepasó la norma y luego fueron llevados a una recta real como intervalos de tiempo, evidencia de eso lo podemos ver en la figura 6.

En el instante que los estudiantes transitan en la fase 4, dentro del mundo matemático, comienzan a vincular conceptos, argumentando de la forma presentada en la figura 7 y siendo ayudados por un pensamiento analítico. Este tipo de pensamiento proviene del momento en que los grupos producen el modelo matemático. Lo analítico está relacionado a descomponer y volver componer los elementos básicos de las inecuaciones lineales, con el fin de poder entender de mejor forma los elementos matemáticos que subyacen en el modelo.

El pensamiento matemático analítico y visual, durante un tiempo han sido desvinculados y menospreciados, debido a que se consideran de poca ayuda en el desarrollo matemático (Zazkis et al., 1996). Otra mirada le ha dado Borromeo-Ferri (2010), al darle importancia en el desarrollo matemático, de forma individual y en conjunto. Según nuestras consideraciones y basados en Zazkis et al. (1996) el pensamiento matemático visual y analítico, deben estar presentes de forma conjunta para desarrollar conceptos matemáticos. Las tareas se pueden construir con un pensamiento matemático más definido que el otro, pero nunca se pueden considerar de forma separada, porque no se podría realizar un desarrollo fundamental de los conceptos matemáticos.

Luego de realizar toda esta ruta de modelación en base a la argumentación y pensamiento matemático analíticovisual, los estudiantes pueden transitar hacia la fase 5 de interpretación a través del tipo de argumentación que da sentido a los procedimientos; pues todo el trabajo matemático realizado en las fases anteriores ayudó a que los estudiantes argumentarán de forma certera, con la acumulación o suma de todas las mediciones de tiempo obtenidas para cada uno de los intervalos que cumplen con el criterio e interpretando este modelo en la vida real (Figura 9).

En relación con la taxonomía de argumentación presentada, sólo se evidenció cuatro de los cinco tipos de argumentación, no porque no surgiera, pues se debe a que no quedó registrado específicamente el momento en que los estudiantes utilizaron el error para realizar una reorganización de las ideas del grupo.

La argumentación en el ciclo de modelación es fundamental, pues en cada fase del ciclo los estudiantes van realizando argumentaciones, debido a que utilizan garantías que se sustentas en conocimientos previos, los cuales se transforman en conclusiones verdaderas. Así sucede con cada tipo de argumentación en la ruta de modelación utilizada: se evidencian conexiones entre conocimientos previos, entre representaciones, se vinculan conceptos, se dan sentido a los procedimientos y se analizan los conceptos erróneos. 


\section{Conclusiones}

La modelación matemática a través de las diferentes tareas de modelación, ayuda a construir conceptos matemáticos desde contextos reales. La realidad que hoy viven los estudiantes determina un cambio de paradigma en la enseñanza-aprendizaje de las matemáticas. La tarea construida para esta investigación, haciendo uso de los elementos de las inecuaciones lineales y nociones física (intensidad del sonido), dio lugar para analizar una canción y cómo esta se puede examinar a partir de las leyes del Ministerio del Medio Ambiente (Ley № 19300, 2011), con relación a ruidos molestos y haciendo uso de un sonómetro.

El tener tareas que nazcan desde una comunidad profesores-investigadores que construyen tareas desde un marco conceptual, ayudan al desarrollo de las matemáticas en los estudiantes. La enseñanza-aprendizaje desde elementos situados y con significado, produce bases sólidas para construir su conocimiento matemático en los estudiantes, pues se erige desde la convergencia de la matemática y su realidad.

Los resultados obtenidos, a través de las producciones de los estudiantes, muestran que es posible realizar cambios en la forma tradicional de enseñanza-aprendizaje de las inecuaciones, pues se soslaya la necesidad de recurrir a procesos algorítmicos para resolver inecuaciones lineales. La idea de comparar funciones establece una forma inicial eficaz para el desarrollo y construcción del objeto matemático.

La utilización de elementos conceptuales inherentes a los objetos matemáticos, ayuda en la construcción conceptual de estos. La comparación es fundamental para comprender y desarrollar las inecuaciones, pues a través de ella se pueden tomar decisiones y delimitar sentencias. Mediante la tarea de modelación presente en esta investigación, los estudiantes desarrollaron un proceso de progreso desde este elemento conceptual transversal, contribuyendo al puente entre las matemáticas y la realidad.

La argumentación y la taxonomía establecida, ayudan a organizar y visualizar como los estudiantes, a través de sus conclusiones, establecen y van dando forma a su conocimiento. A la luz de los resultados y los conocimientos evocados, se establece un precedente sobre la capacidad de los estudiantes para conjeturar en relación con las inecuaciones lineales, cuando estas derivan de situaciones reales.

El modelo, al ser cognitivo, establece dos tipos de estilos de pensamiento matemático, el visual y analítico. Estos dos tipos de estilos de pensamiento matemático realizan una labor muy importante cuando los estudiantes se enfrentan a desarrollar la tarea. Por este motivo, se debe considerar en la construcción de tareas de modelación con una perspectiva cognitiva, la presencia de ambos estilos de pensamiento matemático. Por lo tanto, no consiste sólo en pensamiento matemático visual o pensamiento matemático analítico, sino que las tareas deben construirse desde la mixtura de ambos.

Las tareas de modelación promueven el desarrollo de habilidades y mejora el aprendizaje de las matemáticas, pues el relacionar elementos matemáticos con la realidad, produce en el estudiante un significado de lo que aprende. Además, para los docentes es una ayuda que proporciona bases en donde planificar la construcción y diseño de la enseñanza de un concepto matemático.

La enseñanza-aprendizaje desde elementos situados y la creación de tareas de modelación a través de este medio, debe seguir avanzando en proporcionar tareas al servicio de los docentes, que muchas veces le es difícil relacionar la interacción entre la teoría y el desarrollo de la práctica (Blomhøj, 2019). Además, se debe seguir avanzando en desarrollar la relación dialéctica entre la práctica pedagógica y la investigación (Araújo, 2019), siendo esta investigación una contribución a esta relación dialéctica.

Para futuras investigaciones, se podría usar la misma tarea, pero con un objetivo diferente; específicamente, visualizar las diferentes rutas de modelación utilizadas de forma grupal e individual. En esta investigación sólo se 
realizó el análisis de la ruta tradicional, la cual consiste en ir de fase en fase. Es evidente que la ruta de modelación no es lineal y que tiene diferentes formas de transitar, cuya variación depende de cada grupo e individuo (Borromeo-Ferri, 2010).

La comunidad de educación matemática debe ir avanzando en ir menguando las formas tradicionales de enseñanza-aprendizaje. El Ministerio de Educación en Chile pone a la modelación matemática y la habilidad de argumentar, como elementos principales a desarrollar en los diferentes objetos matemáticos. Esto muestra la necesidad de desarrollar estas habilidades de forma conjunta en las tareas de modelación que se construirán en el futuro.

\section{Referencias bibliográficas}

Araújo, J. (2019). Toward a framework for a dialectical relationship between pedagogical practice and research. In G. A. Stillman \& J. P. Brown (Eds.), Lines of Inquiry in Mathematical Modelling Research in Education (pp. 21-36). Cham: Springer. Doi: https://doi.org/10.1007/978-3-030-14931-4_2

Bagni, R. (2005). Equazioni e disequazioni Riferimenti storici e proprietà interazionali. La matematica e la sua didattica, 3, 285-296. Bologna, Italia: Pitagora.

Barbosa, K. (2003). La enseñanza de inecuaciones desde el punto de vista de la teoría APOE. Revista Latinoamericana de Investigación en Matemática Educativa, RELIME, 6(3), 199-219. Recuperado de https://www.redalyc.org/pdf/335/33560302.pdf

Bernardis, S., Nitti, L. \& Scaglia, S. (2017). Indagación de la historia de las desigualdades matemáticas. Educación matemática, 29(3), 161-187. Doi: https://doi.org/10.24844/em2903.0

Blomhøj, M. (2019). Towards Integration of Modelling in Secondary Mathematics Teaching. In G. A. Stillman \& J. P. Brown (eds.), Lines of Inquiry in Mathematical Modelling Research in Education (pp. 37-52). Cham: Springer. Doi: https://doi.org/10.1007/978-3-030-14931-4_3

Blum, W. \& Leiß, D. (2005). Filling Up'- the problem of Independence-preserving teacher interventions in lessons with demanding modelling tasks. En Bosh, Marianna (Ed.): CERME 4. Proceedings of the Fourth Congress of the European Society for Research in Mathematics Education, pp. 1623-1633.

Borello, M. (2010). Un planteamiento de resignificación de las desigualdades a partir de las prácticas didácticas del profesor. Un enfoque socioepistemológico (Tesis de doctorado no publicada). Centro de Investigación en Ciencia Aplicada y Tecnología Avanzada. Instituto Politécnico Nacional. México. Recuperado de https://repositoriodigital.ipn.mx/handle/123456789/11167

Borromeo-Ferri, R. (2010). On the influence of mathematical thinking styles on learners modeling behavior. Journal für Mathematik-Didaktik, 31(1), pp. 99-118. Doi: https://doi.org/10.1007/s13138-010-0009-8

Cardetti, F. \& LeMay, S. (2019). Argumentation: Building Students' Capacity for Reasoning Essential to Learning Mathematics and Sciences. PRIMUS, 29(8), pp. 775-798. Doi: https://doi.org/10.1080/10511970.2018.1482581

Crespo, C. R.(2005). La importancia de la argumentación matemática en el aula. Premisa, 24, 23-29

De Gamboa, G., Planas, N. y Edo, M. (2010). Argumentación matemática: prácticas escritas e interpretaciones. Suma, 64, 35-44. Recuperado de https://ddd.uab.cat/record/197062 
De Souza, V., Nogueira de Lima, R. \& Campos, T. (2015). A functional graphic approach to inequations. Revista Latinoamericana de Investigación en Matemática Educativa, 18 (1), 109-125. Doi: http://dx.doi.org/10.12802/relime.13.1814.

Duval, R. (1995). Sémiosis et pensée humaine. Registres sémiotiques et apprentissages intellectuels. Neuchâtel, Suisse: Peter Lang.

Ender, E., Fonsi, L. y Yankee, D. (2017). Despacito. En vida [Descarga digital y cencillo en CD]. Los Ángeles: Universal Musica Latino

Frejd, P. \& Bergsten, C. (2018). “Professional Modellers' conceptions of the notion of mathematical modelling: ideas for education". ZDM - The International Journal on Mathematics Education. 50(1-2), 117-127. Doi: https://doi.org/10.1007/s11858-018-0928-2

Garrote, M., Hidalgo, M. y Blanco L. (2004). Dificultades en el aprendizaje de las desigualdades e inecuaciones. Suma, 46, 37 - 44. Recuperado de http://funes.uniandes.edu.co/7228/

Gatica, N. y Maz, A. (2012). Estudio de inecuaciones de dos variables. Actas del XIV Congreso de enseñanza y aprendizaje de las matemáticas. Diversidad y matemáticas. 1-11. Recuperado de http://funes.uniandes.edu.co/21650/1/Gatica2012Estudio.pdf

Halmaghi, E. \& Liljedahl, P. (2012). Inequalities in the history of mathematics: from peculiarities to a hard discipline. In Proceedings of the 2012 annual meeting of the canadian mathematics education study groop/Actes de la recontre annuelle 2012 du groupe canadien d'étude en didactique des mathématiques (pp. 171-178).

Hernández, C. (2013). Consideraciones para el uso del GeoGebra en ecuaciones, inecuaciones, sistemas y funciones. Números. Revista de Didáctica de las Matemáticas, 82, pp. 115-129. Recuperado de http://www.sinewton.org/numeros/numeros/82/Enlared_01.pdf

Kaiser, G. \& Sriraman, B. (2006). "A global survey of international perspectives on modeling in mathematics education". ZDM- The international Journal on Mathematics Education, 38(3), 302-310. Doi: https://doi.org/10.1007/BF02652813

Kemmis, S. y McTaggart, R. (2013). La investigación-acción participativa: La acción comunicativa y la esfera pública. En N. Denzin y Y. Lincoln (coords.). Manual de investigación cualitativa, V. III (pp. 361-439). Barcelona: Gedisa.

León, O. L. y Calderón, D. I. (2001). Validación y argumentación de lo matemático en el aula. RELIME, 4(1), pp. 5-21. Recuperado de http://funes.uniandes.edu.co/9614/1/Leon2001Validacion.pdf

Ley № 19300. Diario oficial de la Republica de Chile, Santiago, Chile, 11 de noviembre de 2012. Recuperado de https://www.bcn.cl/leychile/navegar?idNorma=1040928

MINEDUC (2015). Bases curriculares, 7 mo básico a 2do medio. Santiago, Chile: Unidad de Currículum y Evaluación. Recuperado de https://www.curriculumnacional.cl/614/articles-37136_bases.pdf

MINEDUC (2019). Bases curriculares, 3ro medio y 4to medio. Santiago, Chile: Unidad de Currículum y Evaluación. Recuperado de https://www.curriculumnacional.cl/614/articles-91414_bases.pdf

Monje, Y., Seckel, M. J. y Breda, A. (2018). Tratamiento de la Inecuación en el Curriculum y Textos Escolares Chilenos. Bolema: Boletim de Educação Matemática, 32(61), 480-502. Doi: https://doi.org/10.1590/1980$4415 \mathrm{v} 32 \mathrm{n} 61 \mathrm{a} 09$ 
Muñoz, G., Gutiérrez, V. y Muñoz, S. (2016). Matemática cuarto medio. Texto del estudiante. Santiago: Santillana.

Torres, R. (2013). Aplicación del Enfoque Gráfico en la enseñanza de Inecuaciones: Una. revisión de la experiencia didáctica desde la perspectiva ontosemiótica. El cálculo y su enseñanza, 4, 83-102. Recuperado de http://funes.uniandes.edu.co/14793/1/Torres2013Aplicacion.pdf

Zazkis, R., Dubinsky, E. \& Dautermann, J. (1996). Coordinating visual and analytic strategies: a study of students' understanding of the group D4. Journal for Research in Mathematics Education, 27(4), 435-457. Doi: https://doi.org/10.2307/749876

Esta obra está bajo una Licencia Creative Commons Attribución-NoCommercial 4.0 International

\section{(c) BY-NC}

